\title{
Synthesis of 1,2,3,4,5-pentasubstituted symmetrical pyrroles
}

\author{
G. Ravindran, S. Muthusubramanian*, S. Selvaraj, and S. Perumal \\ Department of Organic Chemistry, School of Chemistry, Madurai Kamaraj University, \\ Madurai-625021, India \\ E-mail: muthumanian2001@yahoo.com
}

\begin{abstract}
The reaction between benzil and 2-[(2-oxo-2-arylethyl)anilino]-1-aryl-1-ethanones is found to yield symmetrical pentasubstituted pyrroles, (5-benzoyl-1,3,4-triphenyl-1H-pyrrol-2yl)(phenyl)methanones in good yield. The structures of the newly synthesized heterocyclic compounds have been established by ${ }^{1} \mathrm{H}$ and ${ }^{13} \mathrm{C}$ NMR spectral data.
\end{abstract}

Keywords: $\alpha$-Diketones, 2-[(2-oxo-2-arylethyl)anilino]-1-aryl-1-ethanone, pyrrole, Hinsberg condensation

\section{Introduction}

Heterocyclic molecules represent the most utilized scaffolds for the discovery of novel synthetic drugs. ${ }^{1}$ As reported in recent communications, the pyrrole moiety can be found both in natural and synthetic pharmaceutical products. ${ }^{2}$ In particular, tetrasubstituted pyrroles have been reported to play an important role as antibacterial, antiviral, anti-inflammatory, and antioxidant agents. ${ }^{3}$ Some combinatorial approaches to pyrrole libraries can be found in the literature. ${ }^{4}$

Paal-Knorr cyclocondensation of 1,4-diketones with amines represents one of the most common approaches to pyrrole synthesis. ${ }^{2 a} 5$ This strategy has also been utilized for the preparation of pyrrole libraries, both in solution ${ }^{6}$ and on the solid phase. ${ }^{7}$ In the Paal-Knorr reaction, the 1,4-dicarbonyl compounds provide the four carbon atoms with their substituents and the amine provides the pyrrole nitrogen with its substituent. The main limitations preventing extensive use of this method are the harsh conditions required for the cyclization and the low availability of properly substituted 1,4-dicarbonyl compounds. A simple method of preparing highly substituted symmetrical pyrroles from symmetrical 1,5-diketones is described in this article. 


\section{Results and Discussion}

We are interested in exploring the synthetic utility of 3-aza analogs of 1,5-diketone, bis phenacyl anilines $\mathbf{1}$. These compounds are not exploited to their full potential and we have used these bis phenacyl anilines to construct several heterocyclic systems like $\mathbf{2}^{8}, \mathbf{3}^{9}$ and $\mathbf{4}^{10}$.

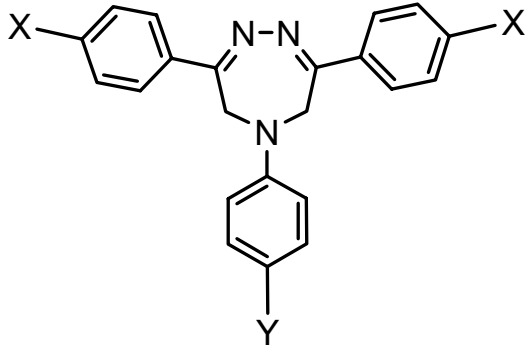

2

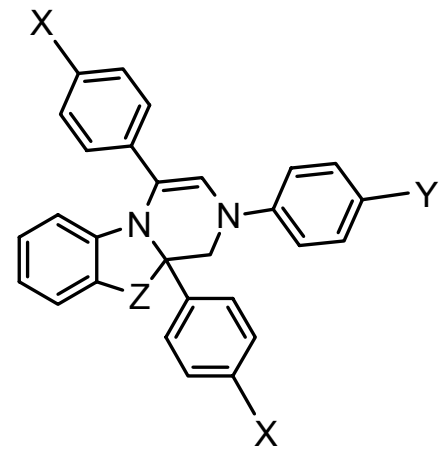

3, $\mathrm{Z}=\mathrm{S}$

The compounds 1 can be easily prepared under solventless condition. ${ }^{8}$ In the present work, the reaction of 2-[(2-oxo-2-arylethyl)anilino]-1-aryl-1-ethanones 1 with benzil in presence of potassium hydroxide in ethanol has been investigated. The reaction affords to (5-benzoyl-1,3,4triphenyl-1H-pyrrol-2-yl)(phenyl)methanones 5 in moderate yield (60-76\%) (Scheme 1).

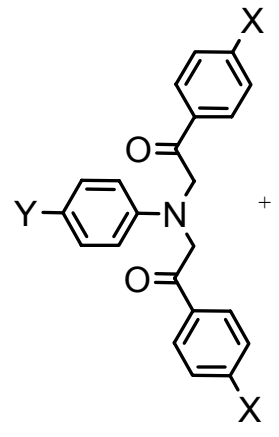

1<smiles>O=C(C(=O)c1ccccc1)c1ccccc1</smiles><smiles>[X]c1ccc(C(=O)c2c(-c3ccccc3)c(-c3ccccc3)c(C(=O)c3ccc([X])cc3)n2C(C)C)cc1</smiles>

5

$\begin{array}{lll}\mathbf{1 , 5} & \mathbf{X} & \mathbf{Y} \\ \mathbf{a} & \mathrm{H} & \mathrm{H} \\ \mathbf{b} & \mathrm{Cl} & \mathrm{H} \\ \mathbf{c} & \mathrm{Me} & \mathrm{H} \\ \mathbf{d} & \mathrm{Cl} & \mathrm{Me} \\ \mathbf{e} & \mathrm{Me} & \mathrm{Me} \\ \mathbf{f} & \mathrm{Me} & \mathrm{OMe}\end{array}$

Scheme 1 
This synthesis of 1,2,3,4,5-pentasubstituted symmetrical pyrroles is similar to Hinsberg condensation. Hinsberg ${ }^{11}$ first described the reaction between benzil and diethyl thiodiacetate to produce the thiophene ring system. It should be mentioned that Hinsberg type condensation has been employed in the construction of pyrrole moieties of different patterns during the syntheses lamellarin, a selective inhibitor of HIV-1 integrase ${ }^{12,13}$ and marine alkaloids. ${ }^{14}$ All the new compounds 5 were characterized by their NMR spectral data.

\section{Experimental Section}

General Procedures. All chemicals were of reagent grade quality and used without further purification. Melting points were measured on a melting point apparatus and are uncorrected. NMR spectra were recorded on a Bruker $300 \mathrm{MHz}$ (Ultrashield) spectrometer. Chemical shifts are reported in ppm relative to tetramethylsilane as an internal standard. The reactions were routinely monitored by thin layer chromatography on silica gel plates.

General procedure for the preparation of (5-benzoyl-1,3,4-triphenyl-1H-pyrrol-2-yl) (phenyl)methanone.A mixture of $1 \mathrm{~g}(3 \mathrm{mmol})$ of benzil and $0.639 \mathrm{~g}(3 \mathrm{mmol})$ of 2-[(2-oxo-2arylethyl) anilino]-1-aryl-1-ethanones 1 in $30 \mathrm{ml}$ of absolute ethanol was heated to near boiling on a steam bath and a solution of $0.340 \mathrm{~g}(6 \mathrm{mmol})$ of potassium hydroxide in $6 \mathrm{ml}$ of absolute ethanol was added in portions. The reaction mixture was heated under reflux for specified time and then cooled to $5^{\circ} \mathrm{C}$ in an ice bath. The dark solid product obtained was filtered under suction and recrystallised from ethanol.

(5-Benzoyl-1,3,4-triphenyl-1H-pyrrol-2-yl)(phenyl)methanone (5a). Yield: 60 \%; Mp: 240 ${ }^{\circ} \mathrm{C}$; Reaction time: $120 \mathrm{~min}$; Anal.calcd.for $\mathrm{C}_{36} \mathrm{H}_{25} \mathrm{NO}_{2}: \mathrm{C}, 85.86 ; \mathrm{H}, 5.00 ; \mathrm{N}, 2.78$; Found: C, 85.83; H, 5.06; N, 2.72. IR (KBr) v cm${ }^{-1}: 3050(\mathrm{C}-\mathrm{H}) ; 1650(\mathrm{C}=\mathrm{O}) .{ }^{1} \mathrm{H}\left(\mathrm{CDCl}_{3}\right) ; 7.11-7.24(\mathrm{~m}$, $6 \mathrm{H}), 7.27-7.33(\mathrm{~m}, 4 \mathrm{H}), 7.43-7.55(\mathrm{~m}, 8 \mathrm{H}), 7.65-7.70(\mathrm{~m}, 2 \mathrm{H}), 7.97-8.05(\mathrm{~m}, 5 \mathrm{H}) ;{ }^{13} \mathrm{C}\left(\mathrm{CDCl}_{3}\right)$; 125.4 , 127.8, 128.0, 128.3 , 128.5, 129.0, 129.3, 129.5, 129.8, 132.9, 133.7, 134.8, 136.4, 139.4, 194.5

[5-(4-Chlorobenzoyl)-1,3,4-triphenyl-1 $H$-pyrrol-2-yl](4-chlorophenyl)methanone

$(5 \mathbf{b})$. Yield: $76 \%$; Mp: $224{ }^{\circ} \mathrm{C}$; Reaction time: 90 min., Anal.calcd.for $\mathrm{C}_{36} \mathrm{H}_{23} \mathrm{Cl}_{2} \mathrm{NO}_{2}$ : C, 75.53; $\mathrm{H}$, 4.05; N, 2.45; Found: C, 75.49; H, 4.07; N, 2.40. IR (KBr) v cm ${ }^{-1}: 3062(\mathrm{C}-\mathrm{H}) ; 1652(\mathrm{C}=\mathrm{O}) .{ }^{1} \mathrm{H}$ $\left(\mathrm{CDCl}_{3}\right) ; 6.90-7.00(\mathrm{~m}, 6 \mathrm{H}), 7.08-7.11(\mathrm{~m}, 2 \mathrm{H}), 7.17-7.30(\mathrm{~m}, 5 \mathrm{H}), 7.49-7.66(\mathrm{~m}, 8 \mathrm{H}), 7.96-8.00$ $(\mathrm{m}, 2 \mathrm{H}) ;{ }^{13} \mathrm{C}\left(\mathrm{CDCl}_{3}\right) ; 127.2,127.3,128.3,128.7,129.4,129.7,130.3,131.0,131.5,132.7$, $133.0,135.3,136.2,139.6,190.0$.

[5-(4-Methylbenzoyl)-1,3,4-triphenyl-1 $H$-pyrrol-2-yl](4-methylphenyl)methanone

(5c). Yield: $70 \%$; Mp: $232{ }^{\circ} \mathrm{C}$; Reaction time: 75 min., Anal.calcd.for $\mathrm{C}_{38} \mathrm{H}_{29} \mathrm{NO}_{2}$ : C, 85.85; H, 5.50; N, 2.63; Found: C, 85.83; H, 5.08; N, 2.62. IR (KBr) $v \mathrm{~cm}^{-1}: 3045(\mathrm{C}-\mathrm{H}) ; 1652(\mathrm{C}=\mathrm{O}) .{ }^{1} \mathrm{H}$ $\left(\mathrm{CDCl}_{3}\right) ; 2.24(\mathrm{~s}, 6 \mathrm{H}), 6.92-6.99(\mathrm{~m}, 10 \mathrm{H}), 7.22-7.25(\mathrm{~m}, 10 \mathrm{H}), 7.55-7.58(\mathrm{~m}, 3 \mathrm{H}) ;{ }^{13} \mathrm{C}\left(\mathrm{CDCl}_{3}\right)$; 
21.6, 126.4, 126.7, 127.6, 128.6, 128.8, 130.0, 130.5, 131.1, 132.1, 133.0, 134.1, 135.1, 139.6, 143.6, 184.3 .

[5-(4-Chlorobenzoyl)-1-(4-methylphenyl)-3,4-diphenyl-1 $H$-pyrrol-2-yl](4-chlorophenyl) methanone (5d). Yield: $72 \%$; Mp: $238{ }^{\circ} \mathrm{C}$; Reaction time: 95 min., Anal.calcd.for $\mathrm{C}_{37} \mathrm{H}_{25} \mathrm{Cl}_{2} \mathrm{NO}_{2}$ : C, 75.77; H, 4.30; N, 2.39; Found: $\mathrm{C}, 75.79 ; \mathrm{H}, 4.35 ; \mathrm{N}, 2.35$. IR (KBr) $v \mathrm{~cm}^{-1}$ : $3055(\mathrm{C}-\mathrm{H}) ; 1653(\mathrm{C}=\mathrm{O}) .{ }^{1} \mathrm{H}\left(\mathrm{CDCl}_{3}\right) ; 2.30(\mathrm{~s}, 3 \mathrm{H}), 6.86-6.92(\mathrm{~m}, 4 \mathrm{H}), 6.94-7.00(\mathrm{~m}, 6 \mathrm{H})$, 7.07$7.11(\mathrm{~m}, 8 \mathrm{H}), 7.57-7.60(\mathrm{~m}, 4 \mathrm{H}) ;{ }^{13} \mathrm{C}\left(\mathrm{CDCl}_{3}\right) ; 21.2,126.4,126.8,127.8,128.2,128.6,129.0$, $130.6,131.1,132.4,132.5,133.2,135.5,135.8,139.2,187.9$.

[5-(4-Methylbenzoyl)-1-(4-methylphenyl)-3,4-diphenyl-1H-pyrrol-2-yl](4-methylphenyl) methanone (5e). Yield: $76 \%$; Mp: $240{ }^{\circ} \mathrm{C}$; Reaction time: 110 min., Anal.calcd.for $\mathrm{C}_{39} \mathrm{H}_{31} \mathrm{NO}_{2}$ : C, 85.84; H, 5.73; N, 2.57; Found: C, 85.83; H, 5.75; N, 2.55. IR (KBr) $\vee \mathrm{cm}^{-1}: 3048(\mathrm{C}-\mathrm{H})$; $1651(\mathrm{C}=\mathrm{O}) .{ }^{1} \mathrm{H}\left(\mathrm{CDCl}_{3}\right) ; 2.24(\mathrm{~s}, 6 \mathrm{H}), 2.27(\mathrm{~s}, 3 \mathrm{H}), 6.92-6.97(\mathrm{~m}, 13 \mathrm{H}), 7.01-7.05(\mathrm{~m}, 5 \mathrm{H})$, 7.56-7.59 (m, $4 \mathrm{H}) ;{ }^{13} \mathrm{C}\left(\mathrm{CDCl}_{3}\right) ; 20.5,20.9,125.7,126.9,127.9,128.9,129.4,130.0,131.2$, $132.0,132.4,133.4,134.4,137.0,139.0,143.0,188.5$.

[1-(4-Methoxyphenyl)-5-(4-methylbenzoyl)-3,4-diphenyl-1 $H$-pyrrol-2-yl](4-methylphenyl) methanone (5f). Yield: $75 \%$; Mp: $226{ }^{\circ} \mathrm{C}$; Reaction time: 90 min., Anal.calcd.for $\mathrm{C}_{39} \mathrm{H}_{31} \mathrm{NO}_{3}$ : C, 83.40; H, 5.56; N, 2.49; Found: C, 83.42; H, 5.52; N, 2.47. IR (KBr) v cm ${ }^{-1}: 3055(\mathrm{C}-\mathrm{H})$; $1650(\mathrm{C}=\mathrm{O}) .{ }^{1} \mathrm{H}\left(\mathrm{CDCl}_{3}\right) ; 2.24(\mathrm{~m}, 6 \mathrm{H}), 3.72(\mathrm{~s}, 3 \mathrm{H}), 6.72-6.74(\mathrm{~m}, 2 \mathrm{H}), 6.87-6.96(\mathrm{~m}, 13 \mathrm{H})$, 7.10-7.13 (m, 3H), 7.55-7.58 (m, $4 \mathrm{H}) ;{ }^{13} \mathrm{C}\left(\mathrm{CDCl}_{3}\right) ; 22.0,55.6,114.4,124.3,126.8,127.7,128.0$, $128.2,129.0,130.4,131.0,133.0,133.5,135.5,139.4,144.1,189.6$.

\section{Acknowledgements}

The authors thank DST, New Delhi for assistance under IRHPA program for the NMR facility.

\section{References}

1. Sperry, J.; Wright, D. Curr. Opin. Drug Discovery Dev. 2005, 8, 723.

2. (a) Gossauer, A. Die Chemie der Pyrrole; Springer-Verlag; Berlin, 1974. (b) Gribble, W. In Comprehensive Heterocyclic Chemistry II; Katritzky, A. R., Rees, C. W., Scriven, E. F. V., Eds.; Pergamon Press: Oxford, 1996, Vol. 2, p 207. (c) Furstner, A. Synlett 1999, 1523. (d) Wiley, J. L.; Compton, D. R.; Dai, D.; Lainton, J. A. H.; Phillips, M.; Huffman J. W.; Martin, B. R. J. Parmacol. Exp. Ther. 1998, 285, 995.

3. (a) Kaiser, D. G.; Glenn, E. M. J. Pharm. Sci. 1972, 61, 1908. (b) Daisone, G.; Maggio, B.; Schillaci, D. Pharmazie 1990, 45, 441. (c) Almerico, A. M.; Diana, P.; Barraja, P.; Dattolo, G.; Mingoia, F.; Putzolu, M.; Perra, G.; Milia, C.; Musiu, C.; Marongiu, M. E. Farmaco 1997, 52, 667. 
4. (a) Dolle. E. R. J. Comb. Chem. 2002, 4, 369. (b) Dolle, E. R. J. Comb. Chem. 2003, 5, 693. (c) Dolle, E. R. J. Comb. Chem. 2004, 6, 623. (d) Dolle, E. R. J. Comb. Chem. 2005, 7, 739.

5. (a) Knorr, L. Chem. Ber. 1885, 18, 299. (b) Paal, C. Chem. Ber. 1885, 18, 367. (c) Sundberg, R. J. In Comprehensive Heterocyclic Chemistry II; Katritzky, A. R., Rees, C. W., Scriven, E. F., Eds.; Pergamon Press: Oxford, 1996; Vol. 2, p 119.

6. Hansford, K. A.; Zanzarova, V.; Dorr, A.; Lubell, W. D. J. Comb. Chem. 2004, 6, 893.

7. Raghavan, S.; Anuradha, K. Synlett. 2003, 711.

8. Ravindran, G.; Muthusubramanian, S.; Selvaraj, S.; Perumal, S. J. Heterocyclic Chem. 2007, 44, 133.

9. Ravindran, G.; Muthusubramanian, S.; Selvaraj, S.; Perumal, S. Phosphorus Sulfur Silicon Relat, Elem. 2007, 182, 509.

10. Ravindran, G.; Muthusubramanian, S.; Selvaraj, S.; Perumal, S. Indian. J. Chem., Sec.B (In Press).

11. Wynberg, H.; Kooreman, H. J. J. Am. Chem. Soc. 1965, 87, 1739.

12. Yamaguchi, T.; Fukuda, T.; Ishibashi, F.; Iwao, M. Tetrahedron Lett. 2006, 47, 3755.

13. Fujikawa, N.; Ohta, T.; Yamaguchi, T.; Fukuda, T.; Ishibashi, F.; Iwao, M. Tetrahedron 2006, 62, 594.

14. Iwao, M.; Takeuchi, T.; Fujikawa, N.; Fukuda, T.; Ishibashi, F. Tetrahedron Lett. 2003, 44, 4443. 